\title{
Closed form optimal exercise boundary of the American put option
}

\author{
Yerkin Kitapbayev*
}

January 12, 2021

\begin{abstract}
We present three models of stock price with time-dependent interest rate, dividend yield, and volatility, respectively, that allow for explicit forms of the optimal exercise boundary of the finite maturity American put option. The optimal exercise boundary satisfies the nonlinear integral equation of Volterra type. We choose time-dependent parameters of the model so that the integral equation for the exercise boundary can be solved in the closed form. We also define the contracts of put type with time-dependent strike price that support the explicit optimal exercise boundary.
\end{abstract}

\section{Introduction}

One of the important problems in mathematical finance is the pricing of American put options. The explicit solution has been obtained in the perpetual case under the classical Black-Scholes model. However, in the finite maturity case the problem has not been solved in the closed form. The main obstacle is the time dependence of the optimal exercise boundary and option price. There were some theoretical characterizations of the option price and optimal exercise boundary in terms of, e.g., integral equation, free-boundary PDE system, series representations etc.

Several numerical approaches have been used to tackle the problem in the case of BlackScholes model. We will mention just some of them. One of the most popular methods in practice is the binomial tree model by Cox, Ross and Rubinstein (1979). Another approach is to reduce the American option pricing problem to a free-boundary PDE system and the latter can be solved, e.g., by the finite difference method (see Brennan and Schwartz (1977)). Then, there was a sequence of papers by Kim (1990), Jacka (1991), and Carr, Jarrow and Myneni (1992), where the so-called early exercise premium (EEP) formula for the American option price has been derived. Based on this result, it was shown that the optimal exercise boundary satisfies the integral equation of Volterra type. Peskir (2005) proved that the optimal boundary is the unique solution to this integral equation. This led to the development of numerical schemes to tackle the corresponding integral equation as it cannot be solved in the closed form. Another strand of the literature was devoted to the exploitation of Monte-Carlo based methods (see

\footnotetext{
*Department of Mathematics, NC State University, Raleigh NC, USA; ykitapb@ncsu.edu

Mathematics Subject Classification 2010. Primary 91G20,60G40. Secondary 60J60, 35R35, 45G10.

Key words and phrases: American put option, geometric Brownian motion, optimal stopping, free-boundary problem, integral equation.
} 
e.g. Longstaff and Schwartz (2001) among others). Finally, several closed form approximations have been introduced (see e.g. Broadie and Detemple (1996), Barone-Adesi and Whaley (1987)).

In this paper, we employ an integral equation approach. The main argument is to deviate from the standard model with constant parameters and choose the deterministic functions for the interest rate (or dividend yield) to obtain the integral equation that can be solved in the closed form. Hence, we provided the extension of the standard Black-Scholes model such that the American put option has an explicit optimal exercise policy. We also specify the extension with time-dependent volatility function that allows us to determine the optimal exercise boundary as the solution to a simple algebraic equation. Finally, we consider the case of the standard model with constant parameters but the contract with time-dependent strike. We select the latter so that the exercise policy can be found in the closed form.

Our paper is somewhat related to the recent work by Kruse and Strack (2019) where socalled inverse optimal stopping problems have been solved. They considered general diffusion framework, and the goal was to determine a time-dependent function $\pi(t)$ that is added to the original payoff in order to obtain the desired stopping rule. Some general existence results and representation formulas for $\pi(t)$ were established. The difference between our paper and Kruse and Strack (2019) is that we mostly seek the model parameters instead of incremental payoff function $\pi(t)$ and also by considering a particular case of American put option under extension of Black-Scholes model we obtained explicit results.

Finally, we will briefly discuss possible applications of the results and directions for future research. One way to employ the representations in this paper is to consider them as the closed form approximations of the American put option price under the standard model. Second, the finite maturity stopping problems can be found in other mathematical finance applications, e.g. defaultable debt pricing, real options, mortgage pricing etc. Due to intractability of finite horizon problems, the arguments of this paper can be used to create examples with closed form solution to these problems. Third, one can try to extend the arguments of this paper to stochastic volatility or jump models with some time-dependent parameters so that explicit optimal exercise rules- can be achieved.

This paper is structured as follows. In Section 2 we review the well-known results for American put option under the standard model with constant parameters. Sections 3, 4, 5 and 6 present expressions for interest rate, dividend yield, volatility and strike functions, respectively, that allow for the optimal exercise boundaries in the closed form.

\section{Standard model: Review}

In this section we briefly review known results for the American put option problem under geometric Brownian motion model with constant parameters. Let us assume that the dynamics of the asset price $X$ under the risk-neutral measure $\mathrm{Q}$ is given by

$$
d X_{t}=(r-\delta) X_{t} d t+\sigma X_{t} d W_{t}
$$

for $t>0$, where $r>0$ is the interest rate, $\delta \geq 0$ is the dividend yield, $\sigma>0$ is the constant volatility, and $W$ is a standard Brownian motion (SBM) under $Q$. It is well known that 
to determine the price $V(t, x)$ of American put option on $X$ at time $t$ with strike $K$ and maturity $T>0$, one can solve the following optimal stopping problem

$$
V(t, x)=\sup _{t \leq \tau \leq T} \mathrm{E}_{t, x}\left[e^{-r(\tau-t)}\left(K-X_{\tau}\right)^{+}\right]
$$

where $\mathrm{E}_{t, x}$ is the expectation under $\mathrm{Q}$ given that $X_{t}=x$, and the supremum is taken over all $\mathcal{F}^{X}$-stopping times $\tau$.

Another well known result is that the optimal exercise policy $\tau^{*}$ can be described by the optimal exercise boundary $b(t)$ such that

$$
\tau^{*}=\inf \left\{s \in[t, T]: X_{s} \leq b(s)\right\} .
$$

Hence, the goal is to find the pair $(V, b)$.

It has been shown in several papers, e.g., Kim (1990), Jacka (1991), Carr, Jarrow and Myneni (1992), that the exercise boundary satisfies the nonlinear integral equation of Volterra type

$$
\begin{aligned}
K-b(t)= & V^{e}(t, b(t)) \\
& +r K \int_{t}^{T} e^{-r(u-t)} N\left(\frac{1}{\sigma \sqrt{u-t}}\left(\log \frac{b(u)}{b(t)}-\left(r-\delta-\frac{\sigma^{2}}{2}\right)(u-t)\right)\right) d u \\
& -\delta b(t) \int_{t}^{T} e^{-\delta(u-t)} N\left(\frac{1}{\sigma \sqrt{u-t}}\left(\log \frac{b(u)}{b(t)}-\left(r-\delta+\frac{\sigma^{2}}{2}\right)(u-t)\right)\right) d u
\end{aligned}
$$

for $t \in[0, T)$ with $b(T-)=K \cdot \min (1, r / \delta)$, where $N$ is the cdf of $\mathcal{N}(0,1)$ and $V_{e}$ is the European option price

$$
\begin{aligned}
V^{e}(t, x)= & K e^{-r(T-t)} N\left(\frac{1}{\sigma \sqrt{T-t}}\left(\log \frac{K}{x}-\left(r-\delta-\frac{\sigma^{2}}{2}\right)(T-t)\right)\right) \\
& -x e^{-\delta(T-t)} N\left(\frac{1}{\sigma \sqrt{T-t}}\left(\log \frac{K}{x}-\left(r-\delta+\frac{\sigma^{2}}{2}\right)(T-t)\right)\right)
\end{aligned}
$$

for $t \in[0, T)$ and $x>0$. Later it was proven by Peskir (2005) that the optimal exercise boundary $b$ is the unique solution to the equation (2.4) in the class of continuous functions. To the best of our knowledge, this equation does not have explicit solution but it can be solved numerically by backward induction and using some quadrature scheme. Once the boundary $b$ is obtained, the American option price can be computed using so-called early exercise premium (EEP) representation formula

$$
\begin{aligned}
V(t, x)= & V^{e}(t, x)+\mathrm{E}_{t, x}\left[\int_{t}^{T} e^{-r(u-t)}\left(r K-\delta X_{u}\right) I\left(X_{u} \leq b(u)\right) d u\right] \\
= & V^{e}(t, x) \\
& +r K \int_{t}^{T} e^{-r(u-t)} N\left(\frac{1}{\sigma \sqrt{u-t}}\left(\log \frac{b(u)}{x}-\left(r-\delta-\frac{\sigma^{2}}{2}\right)(u-t)\right)\right) d u \\
& -\delta x \int_{t}^{T} e^{-\delta(u-t)} N\left(\frac{1}{\sigma \sqrt{u-t}}\left(\log \frac{b(u)}{x}-\left(r-\delta+\frac{\sigma^{2}}{2}\right)(u-t)\right)\right) d u
\end{aligned}
$$


for $t \in[0, T)$ and $x>0$. It essentially decomposes the American option price as the sum of European option price and EEP. The latter takes into the account the local benefits $\left(r K-\delta X_{u}\right)$ of early exercise at time $u$ given that it is optimal to stop, i.e., $I\left(X_{u} \leq b(u)\right)$. Then these benefits are discounted to time $t$ and integrated over the interval $[t, T]$. The second equality follows from Fubini's theorem and the distribution of $X_{u}$.

\section{Time-dependent interest rate}

1. Let us consider American put option problem with strike $K$ and maturity $T$. We assume that the dynamics of the asset price $X$ under the risk-neutral measure $\mathrm{Q}$ is given by

$$
d X_{t}=(r(t)-\delta) X_{t} d t+\sigma X_{t} d W_{t}
$$

with deterministic interest rate $r(t)>0$ (to be specified), constant dividend yield $\delta \geq 0$ and constant volatility $\sigma>0$. The goal of this section is to provide particular form of interest rate that allows for the closed form representation of the optimal exercise rule.

As in the standard model the price of American put can be written as the value function of the stopping problem

$$
V(t, x)=\sup _{t \leq \tau \leq T} \mathrm{E}_{t, x}\left[e^{-\int_{t}^{\tau} r(s) d s}\left(K-X_{\tau}\right)^{+}\right]
$$

for $t \in[0, T)$ and $x>0$.

The analysis below is straightforward extension of the constant interest case so we will focus only on main results. As we have Markovian setting, it is natural to define the exercise and waiting regions, respectively,

$$
\begin{aligned}
& \mathcal{E}=\{(t, x) \in[0, T) \times(0, \infty): V(t, x)=K-x\} \\
& \mathcal{C}=\{(t, x) \in[0, T) \times(0, \infty): V(t, x)>K-x\}
\end{aligned}
$$

Now let us apply Ito-Tanaka's formula for the discounted payoff in order to gain some insight into the structure of the exercise region

$$
\begin{aligned}
\mathrm{E}_{t, x}\left[e^{-\int_{t}^{\tau} r(s) d s}\left(K-X_{\tau}\right)^{+}\right]= & (K-x)^{+}-\mathrm{E}_{t, x}\left[\int_{t}^{\tau} e^{-\int_{t}^{u} r(s) d s} r(u) K I\left(X_{u} \leq K\right) d u\right] \\
& +\mathrm{E}_{t, x}\left[\int_{t}^{\tau} e^{-\int_{t}^{u} r(s) d s} \delta X_{u} I\left(X_{u} \leq K\right) d u\right] \\
& +\frac{1}{2} \mathrm{E}_{t, x}\left[\int_{t}^{\tau} e^{-\int_{t}^{u} r(s) d s} d \ell_{u}^{K}(X)\right]
\end{aligned}
$$

where $\ell^{K}(X)$ is the local time that the process $X$ 'spends' at $K$. The right-hand side can be explained as follows: the first term is the immediate payoff, the second and third terms are respectively the instantaneous losses and benefits (due to postponed interests $r K d u$ and collected dividends $\delta X_{u} d u$, respectively) of waiting to exercise when the option is in-the-money. Hence, one can deduce that there exists the optimal exercise boundary $b(t)$ defined on $[0, T)$ such that

$$
\mathcal{E}=\{(t, x) \in[0, T) \times(0, \infty): x \leq b(t)\} .
$$


2. Now we can apply the same arguments as for the case of constant interest rate to establish EEP representation for the American put price

$$
\begin{aligned}
V(t, x)= & V^{e}(t, x) \\
& +K \int_{t}^{T} e^{-\int_{t}^{u} r(s) d s} r(u) N\left(\frac{1}{\sigma \sqrt{u-t}}\left(\log \frac{b(u)}{x}-\int_{t}^{u}\left(r(s)-\delta-\frac{\sigma^{2}}{2}\right) d s\right)\right) d u \\
& -x \int_{t}^{T} e^{-\delta(u-t)} \delta N\left(\frac{1}{\sigma \sqrt{u-t}}\left(\log \frac{b(u)}{x}-\int_{t}^{u}\left(r(s)-\delta+\frac{\sigma^{2}}{2}\right) d s\right)\right) d u
\end{aligned}
$$

for all $t \in[0, T)$ and $x>0$, where $V_{e}$ is the European option price given as

$$
\begin{aligned}
V^{e}(t, x)= & K e^{-\int_{t}^{T} r(s) d s} N\left(\frac{1}{\sigma \sqrt{T-t}}\left(\log \frac{K}{x}-\int_{t}^{T}\left(r(s)-\delta-\frac{\sigma^{2}}{2}\right) d s\right)\right) \\
& -x e^{-\delta(T-t)} \cdot N\left(\frac{1}{\sigma \sqrt{T-t}}\left(\log \frac{K}{x}-\int_{t}^{T}\left(r(s)-\delta+\frac{\sigma^{2}}{2}\right) d s\right)\right)
\end{aligned}
$$

and $N(\cdot)$ is the cdf of $\mathcal{N}(0,1)$. Now using the continuous pasting condition at $x=b(t)$, we obtain the integral equation for the optimal exercise boundary $b$ in (3.2)

$$
\begin{aligned}
K-b(t)= & V^{e}(t, b(t)) \\
& +K \int_{t}^{T} e^{-\int_{t}^{u} r(s) d s} r(u) N\left(\frac{1}{\sigma \sqrt{u-t}}\left(\log \frac{b(u)}{b(t)}-\int_{t}^{u}\left(r(s)-\delta-\frac{\sigma^{2}}{2}\right) d s\right)\right) d u \\
& -\delta b(t) \int_{t}^{T} e^{-\delta(u-t)} N\left(\frac{1}{\sigma \sqrt{u-t}}\left(\log \frac{b(u)}{b(t)}-\int_{t}^{u}\left(r(s)-\delta+\frac{\sigma^{2}}{2}\right) d s\right)\right) d u
\end{aligned}
$$

for $t \in[0, T)$ with $b(T-)=K \min (1, r(T) / \delta)$.

One can adopt the proof from Peskir (2005) to show that the integral equation (3.9) has a unique solution in the class of continuous functions such that $\delta b(t)-r(t) K<0$ for $t \in[0, T)$. We will now choose the interest rate function $r(t)$ so that $r(T-)>\delta$ (i.e., $b(T-)=K$ ) and the integral equation (3.9) has explicit continuous solution that satisfies $b(t)<\frac{r(t)}{\delta} K$ for $t \in[0, T)$.

Theorem 3.1. Let us assume that the interest rate is given by

$$
r(t)=\frac{n\left(\sqrt{\left(2 \delta+\sigma^{2}\right)(T-t)}\right)}{1+\frac{2 \sigma}{\sqrt{2 \delta+\sigma^{2}}}\left(N\left(\sqrt{\left(2 \delta+\sigma^{2}\right)(T-t)}\right)-\frac{1}{2}\right)} \cdot \frac{\sigma}{\sqrt{T-t}}+\delta+\frac{\sigma^{2}}{2}
$$

for $t \in[0, T)$ and where $n(\cdot)$ is the pdf of $\mathcal{N}(0,1)$. Then the optimal exercise boundary has closed form expression

$$
b(t)=\frac{K}{1+\frac{2 \sigma}{\sqrt{2 \delta+\sigma^{2}}}\left(N\left(\sqrt{\left(2 \delta+\sigma^{2}\right)(T-t)}\right)-\frac{1}{2}\right)}, \quad t \in[0, T) .
$$


Proof. Let us define

$$
\gamma(t)=r(t)-\delta-\sigma^{2} / 2
$$

for $t \in[0, T)$ The main idea is to postulate the particular form of the boundary $b$ such that the integral term in (3.9) can simplified. This can be achieved by selecting

$$
b(t)=K e^{-\int_{t}^{T} \gamma(s) d s}
$$

for $t \in[0, T)$ which implies

$$
N\left(\frac{1}{\sigma \sqrt{u-t}}\left(\log \frac{b(u)}{b(t)}-\int_{t}^{u}\left(r(s)-\delta-\frac{\sigma^{2}}{2}\right) d s\right)\right)=N(0)=\frac{1}{2}
$$

for $u \in[t, T)$.

We can then rewrite the equation (3.9) as follows using integration by parts

$$
\begin{aligned}
& K-K e^{-\int_{t}^{T} \gamma(s) d s}=V^{e}\left(t, K e^{-\int_{t}^{T} \gamma(s) d s}\right)+\frac{K}{2} \int_{t}^{T} e^{-\int_{t}^{u} r(s) d s} r(u) d u \\
& -\delta K e^{-\int_{t}^{T} \gamma(s) d s} \int_{t}^{T} e^{-\delta(u-t)} N(-\sigma \sqrt{u-t}) d u \\
& =K e^{-\int_{t}^{T} r(s) d s} N\left(\frac{1}{\sigma \sqrt{T-t}}\left(\log \frac{K}{b(t)}-\int_{t}^{T}\left(r(s)-\delta-\sigma^{2} / 2\right) d s\right)\right) \\
& -K e^{-\int_{t}^{T}\left(r(s)-\sigma^{2} / 2\right) d s} N\left(\frac{1}{\sigma \sqrt{T-t}}\left(\log \frac{K}{b(t)}-\int_{t}^{T}\left(r(s)-\delta+\sigma^{2} / 2\right) d s\right)\right) \\
& +\frac{K}{2}\left(1-e^{-\int_{t}^{T} r(s) d s}\right) \\
& +K e^{-\int_{t}^{T} \gamma(s) d s} e^{-\delta(T-t)} N(-\sigma \sqrt{T-t})-\frac{K}{2} e^{-\int_{t}^{T} \gamma(s) d s} \\
& -K e^{-\int_{t}^{T} \gamma(s) d s} \int_{t}^{T} e^{-\delta(u-t)} d N(-\sigma \sqrt{u-t}) \\
& =\frac{K}{2} e^{-\int_{t}^{T} r(s) d s}-K e^{-\int_{t}^{T}\left(r(s)-\sigma^{2} / 2\right) d s} N(-\sigma \sqrt{T-t})+\frac{K}{2}\left(1-e^{-\int_{t}^{T} r(s) d s}\right) \\
& +K e^{-\int_{t}^{T} \gamma(s) d s} e^{-\delta(T-t)} N(-\sigma \sqrt{T-t})-\frac{K}{2} e^{-\int_{t}^{T} \gamma(s) d s} \\
& +K e^{-\int_{t}^{T} \gamma(s) d s} \frac{\sigma}{\sqrt{2 \delta+\sigma^{2}}}\left(N\left(\sqrt{\left(2 \delta+\sigma^{2}\right)(T-t)}\right)-\frac{1}{2}\right) \\
& =\frac{K}{2}-\frac{K}{2} e^{-\int_{t}^{T} \gamma(s) d s}+K e^{-\int_{t}^{T} \gamma(s) d s} \frac{\sigma}{\sqrt{2 \delta+\sigma^{2}}}\left(N\left(\sqrt{\left(2 \delta+\sigma^{2}\right)(T-t)}\right)-\frac{1}{2}\right)
\end{aligned}
$$

so that

$$
e^{\int_{t}^{T} \gamma(s) d s}=1+\frac{2 \sigma}{\sqrt{2 \delta+\sigma^{2}}}\left(N\left(\sqrt{\left(2 \delta+\sigma^{2}\right)(T-t)}\right)-\frac{1}{2}\right)
$$


or

$$
\gamma(t)=\frac{n\left(\sqrt{\left(2 \delta+\sigma^{2}\right)(T-t)}\right)}{1+\frac{2 \sigma}{\sqrt{2 \delta+\sigma^{2}}}\left(N\left(\sqrt{\left(2 \delta+\sigma^{2}\right)(T-t)}\right)-\frac{1}{2}\right)} \cdot \frac{\sigma}{\sqrt{T-t}}
$$

for $t \in[0, T)$. Hence, the interest rate must be given as

$$
r(t)=\frac{n\left(\sqrt{\left(2 \delta+\sigma^{2}\right)(T-t)}\right)}{1+\frac{2 \sigma}{\sqrt{2 \delta+\sigma^{2}}}\left(N\left(\sqrt{\left(2 \delta+\sigma^{2}\right)(T-t)}\right)-\frac{1}{2}\right)} \cdot \frac{\sigma}{\sqrt{T-t}}+\delta+\frac{\sigma^{2}}{2} .
$$

We note that $r(t)$ goes to $+\infty$ as $t$ approaches $T$ so that $r(T-)>\delta$ as we assumed at the beginning. Now having expression for $\gamma(t)$, the boundary $b$ is

$$
b(t)=K e^{-\int_{t}^{T} \gamma(s) d s}=\frac{K}{1+\frac{2 \sigma}{\sqrt{2 \delta+\sigma^{2}}}\left(N\left(\sqrt{\left(2 \delta+\sigma^{2}\right)(T-t)}\right)-\frac{1}{2}\right)}
$$

for $t \in[0, T)$.

Remark 3.2. We note that the interest rate function given in (3.10) is increasing in $t$ on $[0, T)$ as the numerator of the first term is increasing in $t$ and the denominator is decreasing.

Remark 3.3. As can be seen from the expression (3.10), the shortcoming of the result is that the interest rate $r(t)$ goes to $+\infty$ as $t$ approaches $T$ (see also left panel of Figure 11). However, if one invests in money market account at $t$, the terminal value at $T$ is still finite

$$
e^{\int_{t}^{T} r(s) d s}<\infty
$$

Despite its unrealistic behavior, this result can be still used as the closed form approximation for the market environment where the interest rate is expected to spike up during the lifetime of an option.

Remark 3.4. If the dividend yield $\delta=0$, then the expressions can be simplified to

$$
r(t)=\frac{\sigma n(\sigma \sqrt{T-t})}{2 \sqrt{T-t} N(\sigma \sqrt{T-t})}+\frac{\sigma^{2}}{2}
$$

and

$$
b(t)=K e^{-\int_{t}^{T} \gamma(s) d s}=\frac{K}{2 N(\sigma \sqrt{T-t})}
$$

for $t \in[0, T)$.

Remark 3.5. We note that when $T$ goes $+\infty$, the optimal exercise boundary converges to the previously known optimal threshold $K /\left(1+\frac{\sigma}{\sqrt{2 \delta+\sigma^{2}}}\right)$ for the perpetual put option when $r=\delta+\sigma^{2} / 2$.

Remark 3.6. Despite the fact that we have found the closed form expression for the exercise boundary $b$ in the chosen model of interest rate, the American option price is still given in the integral form (3.7). 

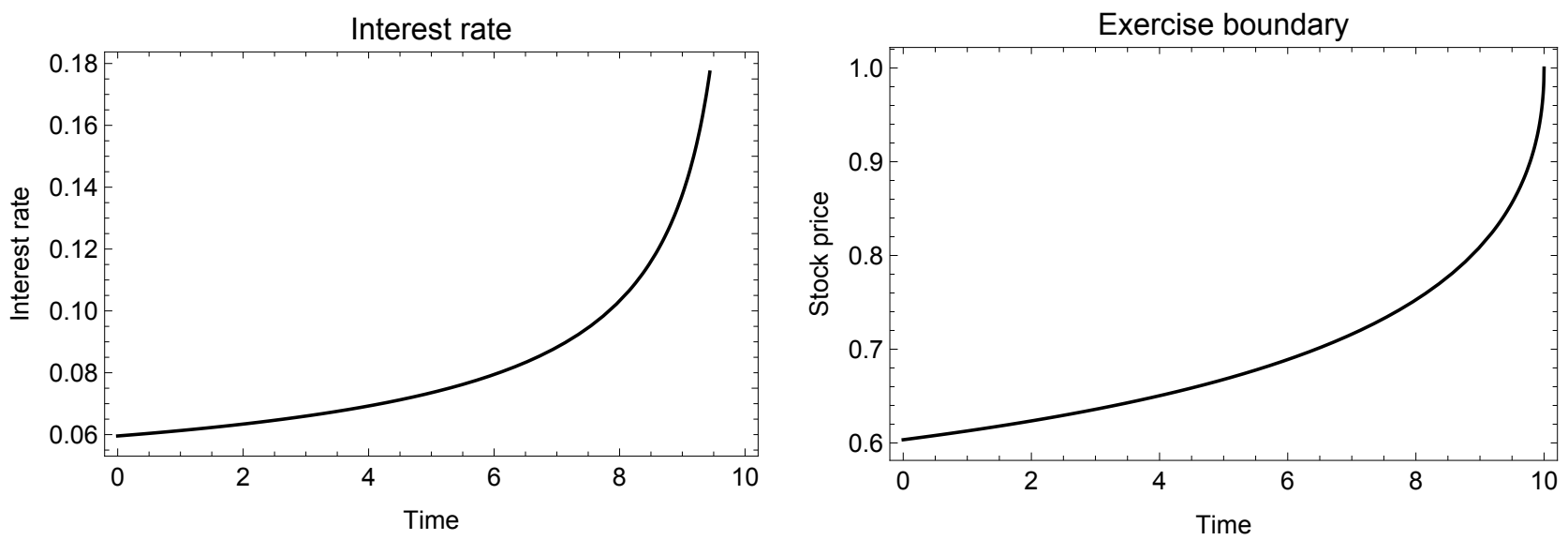

Figure 1: This figure displays the interest rate function (left) given by (3.10) and the optimal exercise boundary (right) given by (3.21). The parameters are $\delta=0, \sigma=0.3, T=10, K=1$.

\section{Time-dependent dividend yield}

1. In this section we derive similar result. The aim again is to offer the model that has closed form expression for the optimal exercise boundary. The difference is that the interest rate is constant now but the dividend yield will be given by particular deterministic function.

We consider American put option problem with strike $K$ and maturity $T$

$$
V(t, x)=\sup _{t \leq \tau \leq T} \mathrm{E}_{t, x}\left[e^{-r(\tau-t)}\left(K-X_{\tau}\right)^{+}\right]
$$

where the asset price $X$ is given by

$$
d X_{t} / X_{t}=(r-\delta(t)) d t+\sigma d W_{t}
$$

with deterministic dividend yield $\delta(t)>0$ (to be specified), constant interest rate $r>0$ and volatility $\sigma>0$, and $W$ is a SBM under the risk-neutral measure $\mathbf{Q}$. The similar arguments from the previous section can be applied here, hence we omit details and highlight only main results and derivations.

2. As in the previous section, there exists the optimal exercise boundary $b$ and the corresponding integral equation can be written as

$$
\begin{aligned}
K-b(t)= & V^{e}(t, b(t)) \\
& +r K \int_{t}^{T} e^{-r(u-t)} N\left(\frac{1}{\sigma \sqrt{u-t}}\left(\log \frac{b(u)}{b(t)}-\int_{t}^{u}\left(r-\delta(s)-\frac{\sigma^{2}}{2}\right) d s\right)\right) d u \\
& -b(t) \int_{t}^{T} e^{-\int_{t}^{u} \delta(s) d s} \delta(u) N\left(\frac{1}{\sigma \sqrt{u-t}}\left(\log \frac{b(u)}{b(t)}-\int_{t}^{u}\left(r-\delta(s)+\frac{\sigma^{2}}{2}\right) d s\right)\right) d u
\end{aligned}
$$

for $t \in[0, T]$ with $b(T-)=K \min (1, r / \delta(T))$, where $V_{e}$ is the European option price

$$
V^{e}(t, x)=K e^{-r(T-t)} N\left(\frac{1}{\sigma \sqrt{T-t}}\left(\log \frac{K}{x}-\int_{t}^{T}\left(r-\delta(s)-\frac{\sigma^{2}}{2}\right) d s\right)\right)
$$




$$
-x e^{-\int_{t}^{T} \delta(s) d s} N\left(\frac{1}{\sigma \sqrt{T-t}}\left(\log \frac{K}{x}-\int_{t}^{T}\left(r-\delta(s)+\frac{\sigma^{2}}{2}\right) d s\right)\right)
$$

for $t \in[0, T)$ and $x>0$.

Now we will select the dividend yield function $\delta(t)$ such that $\delta(T)<r$, i.e., $b(T)=K$ and such that the boundary $b(t)$ has particular form

$$
b(t)=K e^{-\int_{t}^{T} \gamma(s) d s}
$$

for $t \in[0, T)$, where $\gamma(t) \equiv r-\delta(t)+\sigma^{2} / 2$. Note that $\gamma(t)$ here has a different definition from the previous section.

Using this form of the boundary $b$ and integration by parts, we can rewrite the equation (4.3) as follows

$$
\begin{aligned}
& K-K e^{-\int_{t}^{T} \gamma(s) d s}=V^{e}\left(t, K e^{-\int_{t}^{T} \gamma(s) d s}\right)+r K \int_{t}^{T} e^{-r(u-t)} N(\sigma \sqrt{u-t}) d u \\
& -\frac{K e^{-\int_{t}^{T} \gamma(s) d s}}{2} \int_{t}^{T} e^{-\int_{t}^{u} \delta(s) d s} \delta(u) d u \\
& =K e^{-r(T-t)} N\left(\frac{1}{\sigma \sqrt{T-t}}\left(\log \frac{K}{b(t)}-\int_{t}^{T}\left(r-\delta(s)-\sigma^{2} / 2\right) d s\right)\right) \\
& -b(t) e^{-\int_{t}^{T} \delta(s) d s} N\left(\frac{1}{\sigma \sqrt{T-t}}\left(\log \frac{K}{b(t)}-\int_{t}^{T}\left(r-\delta(s)+\sigma^{2} / 2\right) d s\right)\right) \\
& +r K \int_{t}^{T} e^{-r(u-t)} N(\sigma \sqrt{u-t}) d u \\
& -\frac{K e^{-\int_{t}^{T} \gamma(s) d s}}{2}\left(1-e^{-\int_{t}^{T} \delta(s) d s}\right) \\
& =K e^{-r(T-t)} N(\sigma \sqrt{T-t})-\frac{K}{2} e^{-\int_{t}^{T}(\gamma(s)+\delta(s)) d s} \\
& -K e^{-r(T-t)} N(\sigma \sqrt{T-t})+\frac{K}{2} \\
& +K \int_{t}^{T} e^{-r(u-t)} d N(\sigma \sqrt{u-t}) \\
& -\frac{K e^{-\int_{t}^{T} \gamma(s) d s}}{2}\left(1-e^{-\int_{t}^{T} \delta(s) d s}\right) \\
& =\frac{K}{2}-\frac{K e^{-\int_{t}^{T} \gamma(s) d s}}{2}+\frac{\sigma K}{\sqrt{2 r+\sigma^{2}}}\left(N\left(\sqrt{\left(2 r+\sigma^{2}\right)(T-t)}\right)-0.5\right)
\end{aligned}
$$

so that

$$
e^{-\int_{t}^{T} \gamma(s) d s}=1-\frac{2 \sigma}{\sqrt{2 r+\sigma^{2}}}\left(N\left(\sqrt{\left(2 r+\sigma^{2}\right)(T-t)}\right)-0.5\right) .
$$

Taking logarithm and then differentiating both sides, we get

$$
\gamma(t)=\frac{n\left(\sqrt{\left(2 r+\sigma^{2}\right)(T-t)}\right)}{1-\frac{2 \sigma}{\sqrt{2 r+\sigma^{2}}}\left(N\left(\sqrt{\left(2 r+\sigma^{2}\right)(T-t)}\right)-0.5\right)} \cdot \frac{\sigma}{\sqrt{T-t}}
$$


and

$$
\delta(t)=r-\frac{n\left(\sqrt{\left(2 r+\sigma^{2}\right)(T-t)}\right)}{1-\frac{2 \sigma}{\sqrt{2 r+\sigma^{2}}}\left(N\left(\sqrt{\left(2 r+\sigma^{2}\right)(T-t)}\right)-0.5\right)} \cdot \frac{\sigma}{\sqrt{T-t}}+\frac{\sigma^{2}}{2}
$$

for $t \in[0, T)$. We note that $\delta(t)$ goes to $-\infty$ as $t$ approaches $T$ so that $\delta(T-)<r$ as we assumed at the beginning. Then the exercise boundary is given by

$$
b(t)=K\left(1-\frac{2 \sigma}{\sqrt{2 r+\sigma^{2}}}\left(N\left(\sqrt{\left(2 r+\sigma^{2}\right)(T-t)}\right)-0.5\right)\right), \quad t \in[0, T) .
$$

Using the algebraic manipulations we can show that

$$
\delta(t) b(t)-r K<0
$$

for $t \in[0, T)$ and hence we may apply the arguments from Peskir (2005) to show that $b$ given above is the unique solution to (4.3) in the class of continuous functions that satisfy $\delta(t) b(t)-r K<0$ for $t \in[0, T)$.

We can summarize the results of this section as follows.

Theorem 4.1. Let us assume that the dividend yield is given by

$$
\delta(t)=r-\frac{n\left(\sqrt{\left(2 r+\sigma^{2}\right)(T-t)}\right)}{1-\frac{2 \sigma}{\sqrt{2 r+\sigma^{2}}}\left(N\left(\sqrt{\left(2 r+\sigma^{2}\right)(T-t)}\right)-0.5\right)} \cdot \frac{\sigma}{\sqrt{T-t}}+\frac{\sigma^{2}}{2}
$$

for $t \in[0, T)$, then the optimal exercise boundary has closed form expression

$$
b(t)=K\left(1-\frac{2 \sigma}{\sqrt{2 r+\sigma^{2}}}\left(N\left(\sqrt{\left(2 r+\sigma^{2}\right)(T-t)}\right)-0.5\right)\right)
$$

for $t \in[0, T)$.

Remark 4.2. The dividend yield function (4.10) gets negative when $t$ is sufficiently close to $T$ and explodes to $-\infty$ at $T$. This can be the case for the commodities for which the storage costs will rise significantly near the end life of the option. Otherwise, we can make use of these formulas as the closed form approximations for the standard model with constant parameters.

\section{Time-dependent volatility}

In this section we assume that the interest rate is constant, the dividend yield is zero (to simplify analysis) but the volatility will be chosen as particular deterministic function of time in order to have the optimal exercise boundary in simple terms. Unlike in the previous two sections, we do not obtain the closed form for the boundary but there is simple algebraic equation that defines the boundary $b(t)$ for given $t$. Numerically it is still easier to deal with as there is no need to solve the nonlinear integral equation of Volterra type.

Let us consider American put option problem with strike $K$ and maturity $T$

$$
V(t, x)=\sup _{t \leq \tau \leq T} \mathrm{E}_{t, x}\left[e^{-r(\tau-t)}\left(K-X_{\tau}\right)^{+}\right]
$$



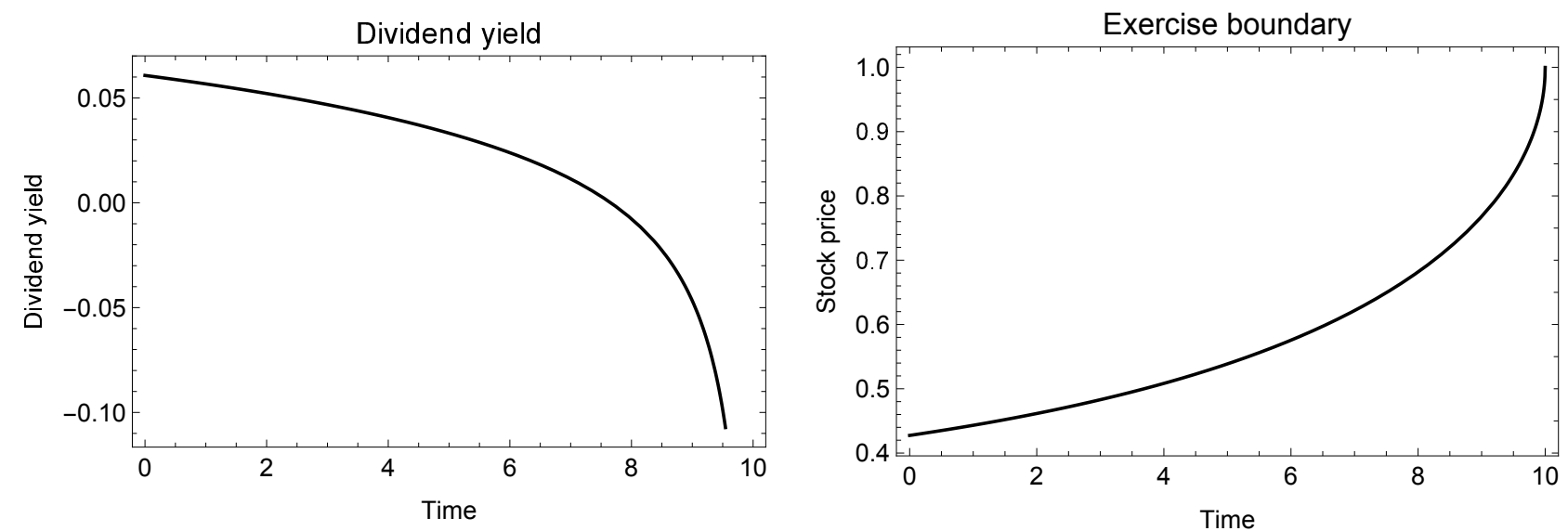

Figure 2: This figure displays the dividend yield function (left) given by (4.10) and the optimal exercise boundary (right) given by (4.11). The parameters are $r=0.05, \sigma=0.3, T=10, K=$ 1.

under the stock price model $X$

$$
d X_{t} / X_{t}=r d t+\sigma(t) d W_{t}
$$

with deterministic volatility $\sigma(t)>0$ (to be specified), constant interest rate $r>0$, and $W$ is a $\mathrm{SBM}$ under $\mathrm{Q}$.

We have the integral equation for the optimal exercise boundary $b$

$$
\begin{aligned}
K-b(t)= & V^{e}(t, b(t)) \\
& +r K \int_{t}^{T} e^{-r(u-t)} N\left(\frac{1}{\sqrt{\int_{t}^{u} \sigma^{2}(u) d u}}\left(\log \frac{b(u)}{b(t)}-\int_{t}^{u}\left(r-\sigma^{2}(s) / 2\right) d s\right)\right) d u
\end{aligned}
$$

for $t \in[0, T]$ with $b(T-)=K$ and this equation has a unique solution in the class of continuous functions such that $b(t)<K$ on $[0, T)$. The European option price $V_{e}$ is given by

$$
\begin{aligned}
V^{e}(t, x)= & K e^{-r(T-t)} N\left(\frac{1}{\sqrt{\int_{t}^{T} \sigma^{2}(u) d u}}\left(\log \frac{K}{x}-\int_{t}^{T}\left(r-\frac{\sigma^{2}(s)}{2}\right) d s\right)\right) \\
& -x N\left(\frac{1}{\sqrt{\int_{t}^{T} \sigma^{2}(u) d u}}\left(\log \frac{K}{x}-\int_{t}^{T}\left(r+\frac{\sigma^{2}(s)}{2}\right) d s\right)\right)
\end{aligned}
$$

for $t \in[0, T)$ and $x>0$. form

$$
b(t)=K e^{-\int_{t}^{T} \gamma(s) d s}, \quad t \in[0, T)
$$


where $\gamma(t)=r-\sigma^{2}(t) / 2$. Using this form of $b$ and integration by parts we obtain

$$
\begin{aligned}
K-K e^{-\int_{t}^{T} \gamma(s) d s}= & V^{e}\left(t, K e^{-\int_{t}^{T} \gamma(s) d s}\right)+\frac{r K}{2} \int_{t}^{T} e^{-r(u-t)} d u \\
= & K e^{-r(T-t)} N\left(\frac{1}{\sqrt{\int_{t}^{T} \sigma^{2}(u) d u}}\left(\log \frac{K}{b(t)}-\int_{t}^{T}\left(r-\sigma^{2}(s) / 2\right) d s\right)\right) \\
& -K e^{-\int_{t}^{T} \gamma(s) d s} N\left(\frac{1}{\sqrt{\int_{t}^{T} \sigma^{2}(u) d u}}\left(\log \frac{K}{b(t)}-\int_{t}^{T}\left(r+\sigma^{2}(s) / 2\right) d s\right)\right) \\
& \left.+\frac{K}{2}\left(1-e^{-r(T-t)}\right)\right) \\
= & \frac{K}{2} e^{-r(T-t)}-K e^{-\int_{t}^{T} \gamma(s) d s} N\left(-\sqrt{\int_{t}^{T}} \sigma^{2}(u) d u\right)+\frac{K}{2}\left(1-e^{-r(T-t)}\right) \\
= & \frac{K}{2}-K e^{-\int_{t}^{T} \gamma(s) d s} N\left(-\sqrt{\int_{t}^{T} \sigma^{2}(u) d u}\right)
\end{aligned}
$$

so that

$$
e^{-\int_{t}^{T} \gamma(s) d s} N\left(\sqrt{\int_{t}^{T} \sigma^{2}(u) d u}\right)=\frac{1}{2}
$$

and hence the volatility function $\sigma(t)$ satisfies the following equation

$$
e^{\int_{t}^{T} \frac{\sigma^{2}(s)}{2} d s} N\left(\sqrt{\int_{t}^{T} \sigma^{2}(u) d u}\right)=\frac{e^{r(T-t)}}{2}
$$

for $t \in[0, T)$. We can rewrite it as

$$
e^{\frac{\phi^{2}(t)}{2}} N(\phi(t))=\frac{e^{r(T-t)}}{2}
$$

where

$$
\phi^{2}(t)=\int_{t}^{T} \sigma^{2}(u) d u
$$

so that

$$
\sigma^{2}(t)=-2 \phi(t) \phi^{\prime}(t)
$$

for $t \in[0, T)$. Thus, $\phi(t)$ can be found as the unique solution $x$ to the algebraic equation

$$
e^{\frac{x^{2}}{2}} N(x)=\frac{e^{r(T-t)}}{2}
$$



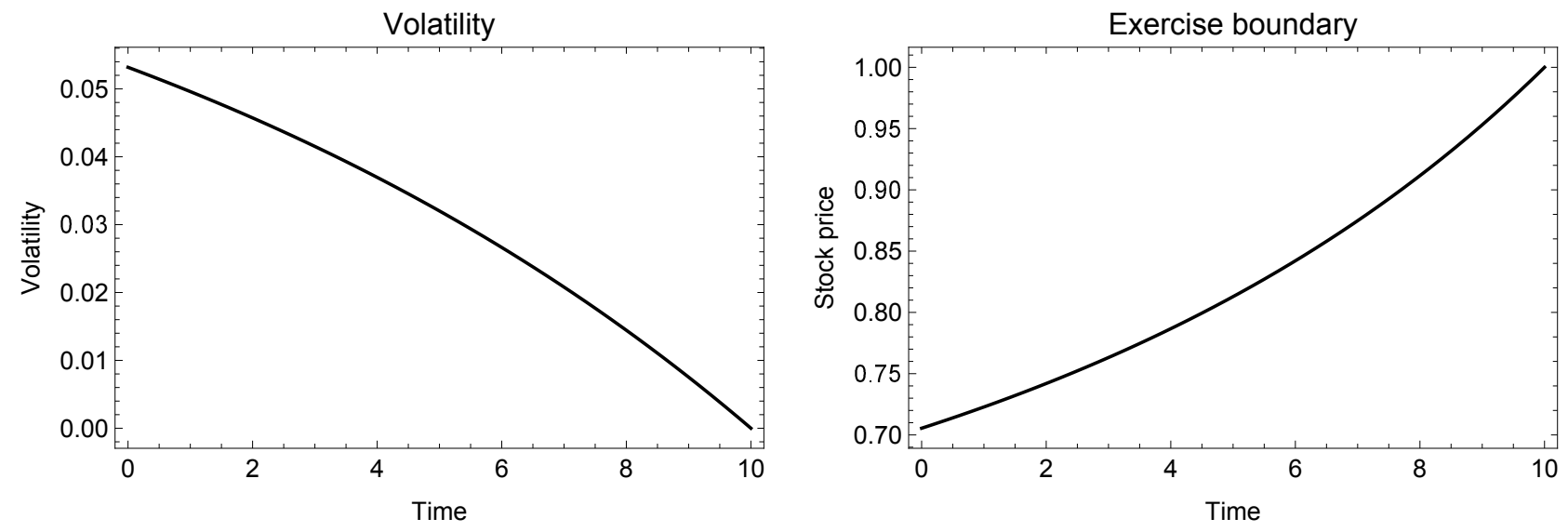

Figure 3: This figure displays the volatility function (left) given by (5.11) and the optimal exercise boundary (right) given by (5.12). The parameters are $r=0.05, T=10, K=1$.

for $t \in[0, T)$. It is clear that $\phi(T-)=0$ and that $\phi$ is decreasing. Once we solve the equation (5.9) for $\phi(t)$, we can determine the volatility $\sigma(t)$ at time $t$ using (5.8). We note that $\sigma(T-)=0$. See Figure 3 for illustrations.

Hence, we derived the following result.

Theorem 5.1. Let us define $\phi(t)$ as the unique solution to the algebraic equation

$$
e^{\frac{\phi^{2}(t)}{2}} N(\phi(t))=\frac{e^{r(T-t)}}{2}
$$

for $t \in[0, T)$. Then if the volatility function is defined as

$$
\sigma(t)=\sqrt{-2 \phi(t) \phi^{\prime}(t)}
$$

for $t \in[0, T)$, the optimal exercise boundary is given by

$$
b(t)=K e^{-r(T-t)} e^{\int_{t}^{T} \frac{\sigma^{2}(s)}{2} d s}=\frac{K}{2 N(\phi(t))}
$$

for $t \in[0, T)$.

Remark 5.2. From numerical experiments we observe that the volatility function given in (5.11) is decreasing on $[0, T)$ with $\sigma(T-)=0$. This can be appropriate for situations where there is a gradual learning about the asset dynamics and hence lower uncertainty over time. Time $t=0$ corresponds to a peak of volatility, for example, after the earnings announcement, and then volatility decreases on $[0, T)$ with extremely low values at $T$.

\section{Time-dependent strike}

1. In this section, we have the standard model with constant parameters. But we consider American put option with time-dependent strike $K(t)$ (to be specified) so that the optimal exercise boundary is given in the closed form. In other words, we stay in the Black-Scholes 
model but aim to choose the contract of put type with the explicit optimal exercise policy. The problem can be formulated as

$$
V(t, x)=\sup _{t \leq \tau \leq T} \mathrm{E}_{t, x}\left[e^{-r(\tau-t)}\left(K(\tau)-X_{\tau}\right)^{+}\right]
$$

for $t \in[0, T)$ and $x>0$, where the asset price $X$ follows

$$
d X_{t}=r X_{t} d t+\sigma X_{t} d W_{t}
$$

with constant interest rate $r>0$ and volatility parameter $\sigma>0$.

Again, the structure of the problem is similar to previous settings. There exists the optimal exercise boundary $b$. However, in this case, the local benefits of waiting to exercise are given as

$$
K^{\prime}(t)-r K(t)
$$

for $t \in[0, T)$. If we wait at time $t$ and do not exercise, the increase $K^{\prime}(t) d t$ in the strike price is instantaneous gain but there are postponed interest payments $r K(t)$ on $K$.

Using standard arguments we derive the integral equation for the boundary $b$

$$
\begin{aligned}
K(t)-b(t)= & V^{e}(t, b(t)) \\
& +\int_{t}^{T} e^{-r(u-t)}\left(r K(u)-K^{\prime}(u)\right) N\left(\frac{1}{\sigma \sqrt{u-t}}\left(\log \frac{b(u)}{b(t)}-\left(r-\frac{\sigma^{2}}{2}\right)(u-t)\right)\right) d u
\end{aligned}
$$

for $t \in[0, T]$, where $V^{e}$ is the price of European put option with strike $K(T)$ under standard Black-Scholes model with constant parameters.

As in the previous sections, we postulate the particular form for $b$ that makes the probability $\mathrm{Q}\left(X_{u} \leq b(u)\right)$ to be 0.5 . This can be achieved by

$$
b(t)=K(T) e^{-\gamma(T-t)}
$$

for $t \in[0, T)$, where $\gamma=r-\sigma^{2} / 2$. We can then determine the strike function $K(t)$ that supports the expression for the boundary $b$ by rewriting the integral equation as follows

$$
\begin{aligned}
K(t)-K(T) e^{-\gamma(T-t)}= & V^{e}\left(t, K(T) e^{-\gamma(T-t)}\right) \\
& +\frac{1}{2} \int_{t}^{T} e^{-r(u-t)}\left(r K(u)-K^{\prime}(u)\right) d u \\
= & \frac{1}{2} K(T) e^{-r(T-t)}-K(T) e^{-\gamma(T-t)} N(-\sigma \sqrt{T-t}) \\
& -\frac{1}{2} K(T) e^{-r(T-t)}+\frac{1}{2} K(t) \\
= & -K(T) e^{-\gamma(T-t)} N(-\sigma \sqrt{T-t})+\frac{1}{2} K(t)
\end{aligned}
$$

so that

$$
K(t)=2 K(T) e^{-\gamma(T-t)} N(\sigma \sqrt{T-t})
$$




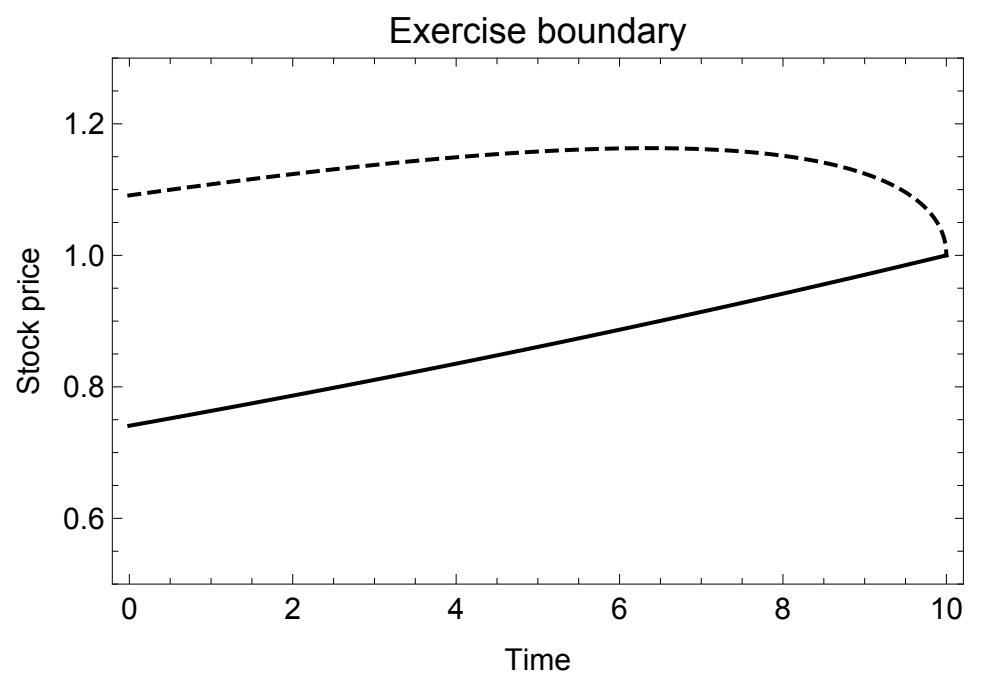

Figure 4: This figure displays the strike function $K(t)$ (dashed) given by (6.6) and the optimal exercise boundary $b(t)$ (solid) given by (6.5). The parameters are $r=0.05, \sigma=0.2, T=$ $10, K(T)=1$.

for $t \in[0, T)$. We can then choose any value of $K(T)$ so that we will obtain closed form expressions for $b(t)$ and $K(t)$ given by (6.5) and (6.6), respectively. Figure 4 displays these results for given set of parameters. We note that the boundary $b$ is increasing (respectively, decreasing) if $r>\sigma^{2} / 2$ (respectively, $r<\sigma^{2} / 2$ ).

2. Now we will add some flexibility when define the boundary $b$. Let us assume the particular form

$$
b(t)=K(T) e^{-\gamma(T-t)}, \quad t \in[0, T)
$$

where $\gamma=m+r-\sigma^{2} / 2$ and $m$ is some constant that we can choose. After some tedious algebra, we can derive the linear integral equation of Volterra type for $K(t)$

$$
\begin{aligned}
K(t)= & 2 K(T) e^{-\left(m+r-\sigma^{2} / 2\right)(T-t)} N\left(-\left(\frac{m}{\sigma}-\sigma\right) \sqrt{T-t}\right) \\
& +\frac{m}{\sigma} \int_{t}^{T} e^{-\left(r+\frac{m^{2}}{2 \sigma^{2}}\right)(u-t)} K(u) \frac{d u}{\sqrt{u-t}}
\end{aligned}
$$

for $t \in[0, T)$. Hence, given some $m$ we find corresponding $K(t)$ by solving the linear Volterra equation above, which is simpler than the standard nonlinear Volterra equation of the second kind for $b(t)$. It can be solved numerically faster than the equation for $b$. See Figure 5 for illustrations.

Thus there is a trade-off here: if one wants to have some freedom by controlling the rate $m$, the linear integral equation for $K(t)$ must be solved numerically, and the closed form $K(t)$ exists only if $m=0$. 


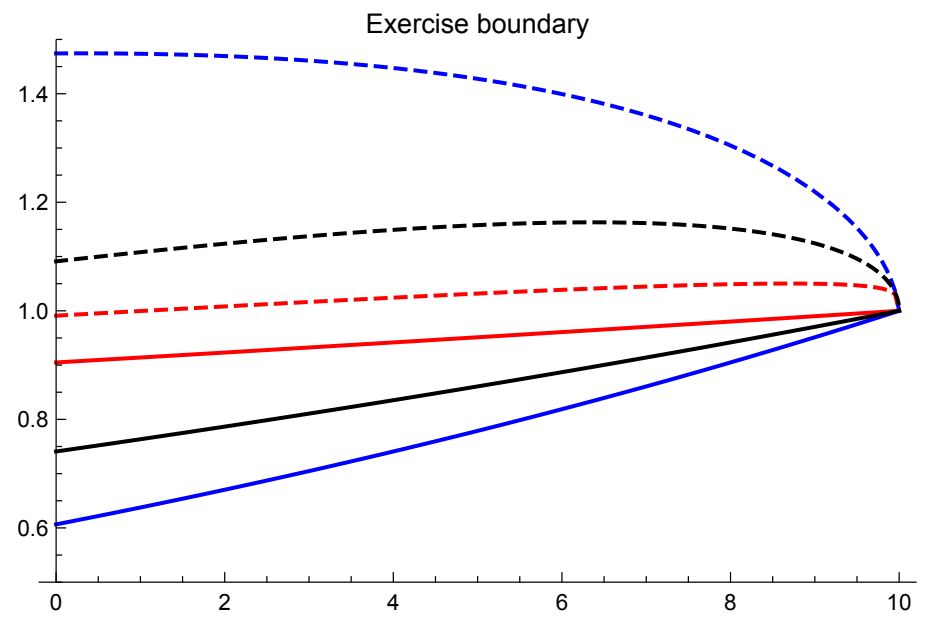

Figure 5: This figure displays the strike function $K(t)$ (dashed) and the optimal exercise boundary $b(t)$ (solid) for different values of parameter $m: m=-0.02$ (red), $m=0$ (black), and $m=0.02$ (blue). The parameters are $r=0.05, \sigma=0.2, T=10, K(T)=1$.

\section{References}

Barone-Adesi, G. and Whaley, R. (1987). Efficient analytic approximation of American option values. J. Finance 42 (301-320).

Brennan, M. and Schwartz, E. (1977). The valuation of American put options. J. Finance 32 (449-642).

Broadie, M. and Detemple, J. (1996). American option valuation: new bounds, approximations, and a comparison of existing methods. Rev. Financial Stud. 9 (1211-1250).

Carr, P., Jarrow, R. and Myneni, R. (1992). Alternative characterizations of American put options. Math. Finance 2 (78-106).

Cox, J., Ross, S. and Rubinstein, M. (1979). Option pricing: a simplified approach, J. Financ. Econ. 7 (229-263).

Kruse, T. and Strack, P. (2019). An inverse optimal stopping problem for diffusion processes. Math. Oper. Res. 44 (423-439).

Kim, I. (1992). The analytic valuation of American options. Rev. Financial Stud. 3 (547-572).

Jacka, S. (1991). Optimal stopping and the American put. Math. Finance 1 (1-14).

Longstaff, F. and Schwartz, E. (2001). Valuing American options by simulation: a simple leastsquares approach Rev. Financial Stud. 14 (113-147).

Peskir, G. (2005). On the American option problem. Math. Finance 15 (169-181). 